\title{
Digital beamform simulation in anechoic chamber
}

\author{
Elena Dobychina* and Mikhail Snastin \\ Moscow Aviation Institute (National Research University) "MAI", 125993 Moscow, Volokolamskoe \\ shosse, 4, Russia
}

\begin{abstract}
The results of digital beamform simulation, principles of processing and use of the obtained experimental data are presented. Methods were developed and an experimental setup was created for fullscale modeling and measurement of the characteristics of digital antenna systems with spatio-temporal signal processing at the Scientific and Production Center for Radio Information Metrology of the Moscow Aviation Institute (SPC RIM MAI).
\end{abstract}

\section{Introduction}

Digital antenna array (DAA) is an antenna system, which is a set of analog-digital channels in which the radiation pattern is formed in digital form, without phase shifters [1]. Modern technologies of DAA owe their massive development to the integration of digital signal processing processors with analog-to-digital and digital-to-analog converters within a single module [2]. The construction of DAA channels on such a basis makes it possible to unify procedures and hardware nodes of signal processing and simplifies their adaptation to a particular operating protocol. Moreover, the architecture of electronic equipment can be optimized for resources and functionality for directly performed tasks [3].

A key feature of the DAA is the digital beamforming of the radiation pattern of the antenna array. A multiple antenna beams, synthesized, for example, using fast Fourier transform algorithms or through classical discrete Fourier analysis procedures, is a set of spatial-frequency filters, each of which selects a strictly defined set of signals and suppresses the rest, perceived as interference.

Full-scale modeling of digital diagram formation (DDF) processes, principles of processing and use of the obtained experimental results is the most important stage in the practical implementation of a new type of antenna systems - DAA.

\section{Object and experimental technique}

An experimental setup has been assembled in the antenna measuring and computing complex based on an anechoic chamber, which allows reproducing the process of digital diagram formation (Fig 1). The studies were discrete measurements of the complex transmission coefficient using a vector network analyzer (VNA) N 5225 A between the QR

* Corresponding author: dem5577@gmail.com 
2000 transmitting antenna and each element of the receiving linear array, which was modeled by linear displacement along the X axis of the SH 4000 or SH 800 measuring probe on the planar antenna positioner scanner [4].

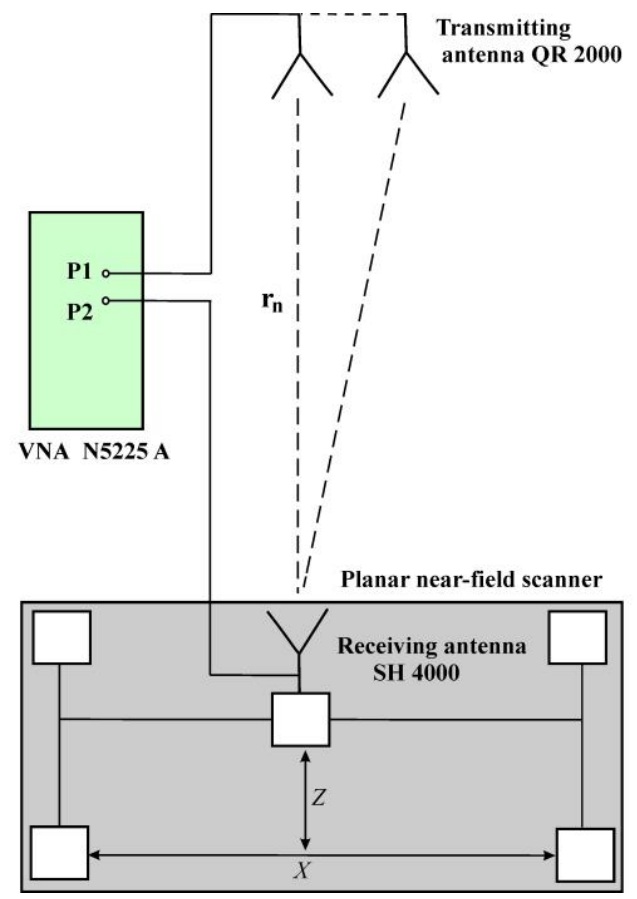

Fig. 1. Schematic of the experimental system in anechoic chamber.

An example of the location of the SH 4000 antenna on the positioner of a planar scanner is shown in Fig.2.

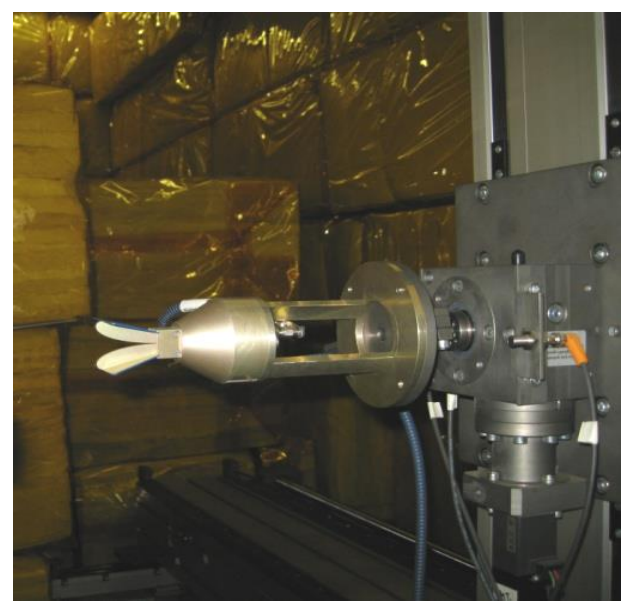

Fig. 2. Antenna SH 4000 on the scanner.

A radiator simulating a direction-finding target was installed in the far zone of a virtual receiving digital antenna array [5]. The transmitting antenna, as a target simulator, was installed opposite the central position of the receiving digital aperture element, i.e. the center position of the antenna on a planar movable scanner. Before this, a full two-port SOLT calibration was carried out at each measured frequency. Measurements were 
repeated for several transmitter power levels and different receiver bands; vertical polarization was established.

\section{The results of experimental studies and analysis of the data}

At the first stage, the operating frequency $f=3 \mathrm{GHz}$ was selected, the receiving antenna on the scanner moved with a step equal to $\lambda / 2$, having traveled a distance of $40 \mathrm{~cm}$. The far zone for the antenna array with similar parameters is $320 \mathrm{~cm}$, and in the experiment a radiating antenna simulating the target was located more than $325 \mathrm{~cm}$ from the scanner.

As a result of measurements, samples of the amplitude and phase distribution along the plane of the virtual antenna were obtained, presented respectively in Fig. 3 and Fig. 4.

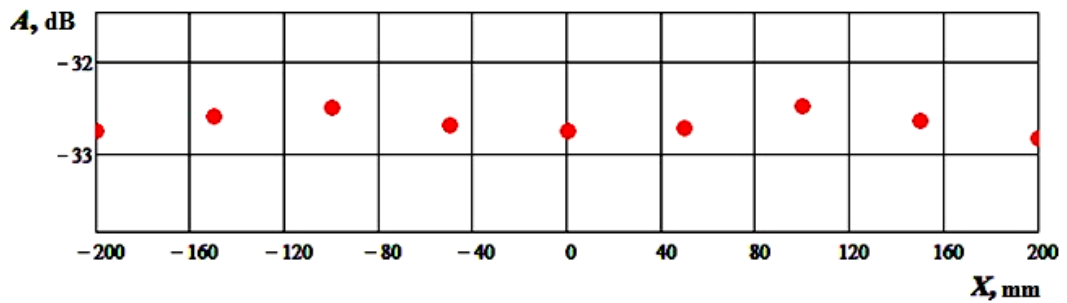

Fig. 3. Sample amplitude distribution of the digital antenna array.

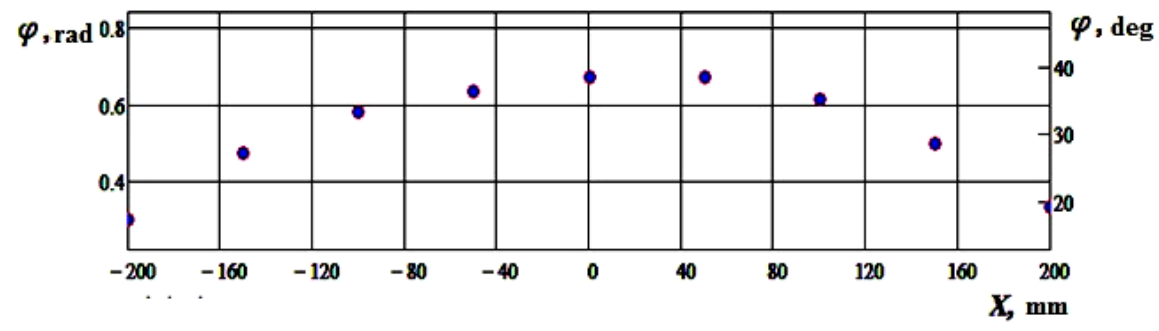

Fig. 4. Sample phase distribution of the digital antenna array.

Using a special algorithm, it is possible to reconstruct the digital total radiation pattern of the model of the experimental digital array (Fig. 5), the maximum of which corresponds to the angle of arrival of the signal from the antenna of the target simulator.

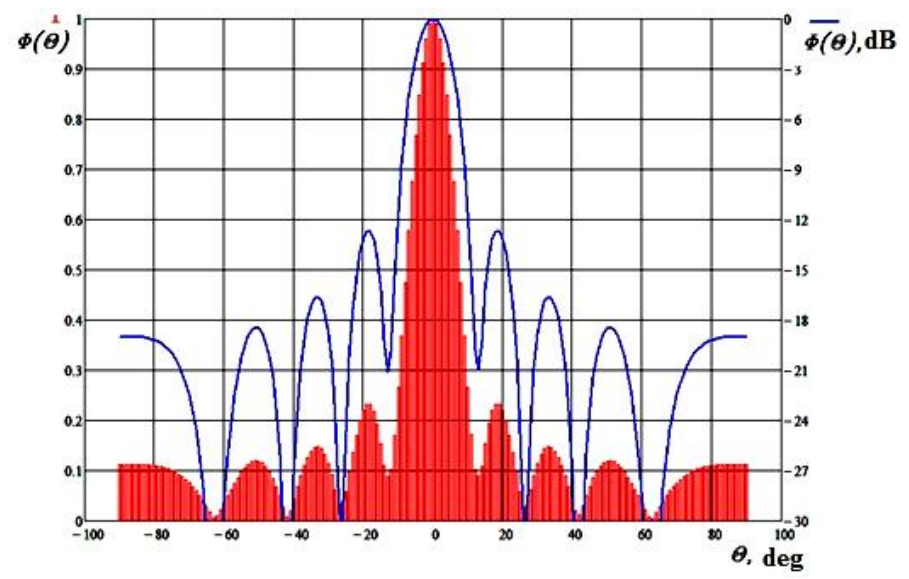

Fig. 5. The directional pattern of experimental DAA, the angle of arrival is 0 degrees. 
With digital processing in the antenna array, it is not difficult to construct a difference radiation pattern without additional measurements, which is shown in Fig. 6.

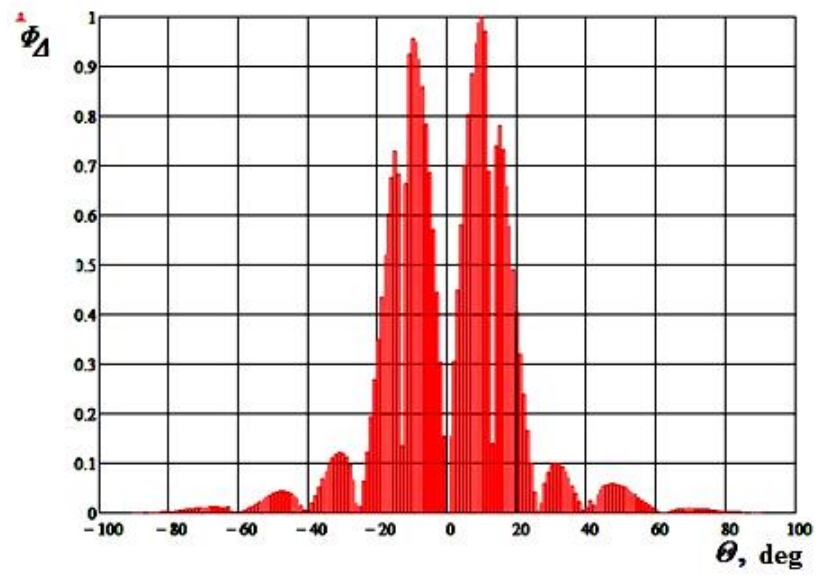

Fig. 6. The differential radiation pattern of the experimental DAA.

The digital method for calculating the radiation pattern makes it possible to increase the accuracy of determining the angle of arrival of the signal from the simulator without additional tools, only by changing the parameters of the calculation algorithm [5]. An example of calculating a digital radiation pattern with a smaller angular pitch is shown in Fig. 7 and Fig. 8.

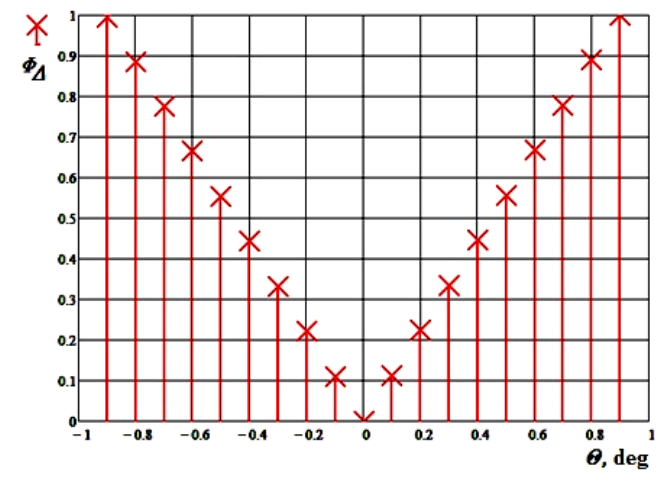

Fig. 7. The differential radiation pattern of the experimental DAA, sample discretion $0.1 \mathrm{deg}$.

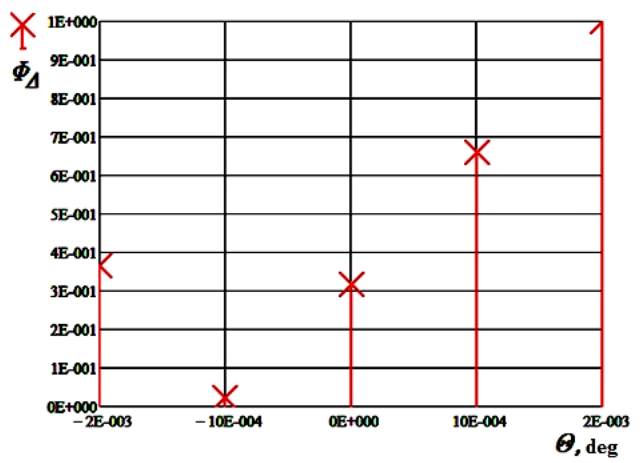

Fig. 8. The differential radiation pattern of the experimental DAA, sample discretion $0.01 \mathrm{deg}$. 
If the amount of computational cost is not a limitation in a specific DAA, then theoretically the direction finding error can be reduced to arbitrarily small values. This is illustrated by the difference radiation pattern (Fig. 8), where the calculation was performed in increments of 0.01 degrees. As a result, it was possible to detect the angular deviation of the radiation source from the normal by 0.01 degrees.

\section{Conclusion}

An algorithm is compiled and an example of the formation of a digital radiation pattern using a simulated full-scale model of the receiving DAA is shown. The possibility of digital electric scanning in space and high-precision determination of the angular coordinate of the target is confirmed. The measurements were carried out in the presence of noise arising even in anechoic chambers due to the limited reflection of the signal from closely located objects intentionally used in the presented experiment.

A series of experiments was carried out in a shielded anechoic chamber using a planar antenna scanning unit, as a result of which the total and difference digital radiation patterns of the DAA simulation model were fully digitally constructed, and the direction-finding mode of the radiation source was reproduced with the determination of the angle of arrival of the signal accurate to thousandths of the width of the radiation pattern antenna array.

The report was prepared based on the results of a study carried out as part of the implementation of the state order "Ensuring the conduct of scientific research" at the Scientific and Production Center for Radio Information Metrology (SPC RIM) of the Moscow Aviation Institute.

\section{References}

1. L.N. Grigoriev, Digital beamforming in phased array antennas (Radioengineering, 2010)

2. E.M. Dobychina, Yu.V. Koltsov, Digital antenna arrays in board radar systems (Publisher MAI, 2013)

3. E.M. Dobychina, P.A. Shmachilin, The construction of digital antenna arrays for modern radioelectronic systems (Radioengineering, 2014)

4. S. Gregson, J. McCormick, C. Parini, Principles of Planar Near-Field Antenna Measurements (IET, 2007)

5. E.M. Dobychina, M.V. Snastin, S.V. Kharalgin, Investigation of a digital antenna array in a shielded anechoic chamber, Scientific and Technical Volga region Bulletin, v. 12, pp.136-139 (2018) 